\title{
Intensity and number of alternatives in hue identification: Piéron's law and choice reaction time
}

\author{
RICHARD SCHWEICKERT, CYNTHIA DAHN, and KIMBERLY MCGUIGAN \\ Purdue University, West Lafayette, Indiana
}

\begin{abstract}
Two experiments investigated the effects of intensity and the number of response alternatives in hue identification. Three questions were addressed. First, what is the relationship between these effects? If intensity and number of alternatives affect different processes in series, these factors would have additive effects on reaction time (RT). Other models predict a multiplicative interaction. These models assume that when a stimulus is presented, evidence accrues over time for each response alternative, and that when the evidence for a particular alternative exceeds its criterion, the response is made. A natural auxiliary assumption is that intensity affects the rate of evidence accrual, while the number of alternatives affects the criterion. The second question addressed is, how does choice RT change as a function of intensity? Piéron used a power function to describe the effect of intensity on simple RT. This paper considers three candidates for intensity's effect on choice RT: the power, logistic, and Michaelis functions. Each candidate function was tested in an additive model and a multiplicative model. The best account was given by the power function in an additive model. The last question is, does each process produce an output that can be classified as correct or incorrect? Schweickert (1985) proposed that factors that selectively influenced such processes would have additive effects on log percent correct. Instead, an interaction was found. Perhaps the assumption that the process affected by intensity produces a single output is wrong.
\end{abstract}

Stimulus intensity in cognitive tasks is often investigated because it is assumed to demarcate the processing that occurs immediately after stimulus presentation. Its effects on reaction time are usually found to be additive with the effects of other factors, such as foreperiod duration, similarity, stimulus-response compatibility, and practice (Everett, Hochhaus, \& Brown, 1985; Niemi, 1979; Raab, Fehrer, \& Hershenson, 1961; Sanders \& Andriessen, 1978; Shwartz, Pomerantz, \& Egeth, 1977). A usual explanation for additivity is that intensity and the other factors affect different processes arranged in series (Fisher \& Goldstein, 1983; Schweickert, 1978; Sternberg, 1969). In this paper we report on the effects of intensity in relation to the effects of the number of alternative responses, factors already found to have additive effects in previous experiments (Niemi, 1979; Posner, 1978, p. 114; Smith, 1967). We will call the process affected by intensity, en-

\footnotetext{
This research was supported by NIMH Grants MH38675 and MH41452. We thank Brian Boruff and Cathrin Hayt for help with the experiments, and Alex Cherry Wilkinson for advice about hardware. Software for running the experiments was written by Robert Macmillian, George Adams, and David Tumenoksa. Barry Kantowitz generously lent his lab and equipment. Jerome R. Busemeyer, Philip Smith, and Gerald S. Wasserman provided very helpful suggestions. Special thanks are due to Leslie Kane for many contributions, especially in data analysis. Patrick Graham kindly accommodated his schedule to allow time for revisions. Correspondence may be addressed to Richard Schweickert, Department of Psychological Sciences, Purdue University, West Lafayette, IN 47907.
}

coding. We will call the process prolonged by increasing the number of alternatives, the decision, leaving open the question of whether this is the same process as that affected by intensity.

Additivity between intensity and the number of alternatives is surprising because intensity is known to affect the rate of sensory impulses, and it would seem that the speed of the decision would vary with the rate of the impulses (Brebner \& Welford, 1980). A broad class of models (variable sampling models) assumes that evidence for each response alternative accrues over time, and that when the evidence for some alternative exceeds its criterion, that response is made (Grice, 1968; Luce, 1986; McGill, 1963; Nissen, 1977; Vickers, Burt, Smith, \& Brown, 1985; Vickers, Smith, Burt, \& Brown, 1985). An approximate value for the expected time required for the decision according to such models is $d=b / a$, where the criterion is set at $b$ and the rate at which evidence accrues for a particular alternative is $a$ (Vickers, Smith, et al., 1985 , p. 190). This equation is only approximate since it ignores errors (Link, 1979; Townsend \& Ashby, 1983, p. 275). It also ignores the fact that if the evidence is discrete, the criterion will be overshot by a little rather than hit exactly. Other methodological issues are discussed in Busemeyer and Jones (1983).

Variable sampling models in themselves are not ruled out by additivity between intensity and the number of alternatives, because if each factor is allowed to affect each of the parameters, $a$ and $b$, then the models can be made 
to predict additivity. But a natural auxiliary assumption is that decreasing the intensity lowers the rate $a$, whereas increasing the number of alternatives raises the criterion $b$. Then the decision time when intensity is at level $i$ and the number of alternatives is at level $j$ is $d_{i j}=b_{j} / a_{i}$. If other discrete stages precede and follow the decision, let $r$ be the mean time required for them. Then the expected reaction time would be

$$
t_{i j}=b_{j} / a_{i}+r .
$$

This predicts an overadditive interaction,

$$
t_{22}-t_{21}-t_{12}+t_{11}=\left(a_{1}-a_{2}\right)\left(b_{2}-b_{1}\right) / a_{1} a_{2}>0,
$$

where for each factor, the higher subscript indicates the more difficult level.

Although this prediction is based on an approximation, very special assumptions would have to be made about the quantities ignored in order to compensate exactly for the overadditivity in the equation above. Note also that if there are several stimuli, and if each has its own accrual rate and criterion, there will be an overadditive interaction for each stimulus considered separately. If the experiment is designed so that each stimulus is presented equally often in each condition over the course of the experiment, then the interaction for the overall mean reaction time will be the mean of several overadditive interactions, and will be overadditive.

In previous experiments investigating intensity in relation to the number of alternatives, the stimuli have been different digits (Posner, 1978) or locations on the left or right (Niemi, 1979; Smith, 1967). A property that might be more conducive to producing an interaction is hue. According to Garner (1974), hue and intensity are integral dimensions, and because of this special relationship they are processed together.

\section{The Intensity Function}

The effects of intensity on simple reaction time were described by Piéron $(1914,1920$; see also Kohfeld, Santee, \& Wallace, 1981) with a power function,

$$
t=r+b / I^{p}
$$

where $I$ is the intensity of the stimulus. This suggests that when the onset of the stimulus is critical for the response, the rate of accrual of evidence $a(I)$ is a power function,

$$
a(I)=\left(I-I_{0}\right)^{p},
$$

where $I_{0}$ is the threshold intensity. Would a power function be suitable when the subject must identify the stimulus, rather than merely detect its onset?- Two other candidate functions were also considered. A function often proposed as relating stimulus intensity and response output is the Michaelis function,

$$
a(I)=\frac{I^{p}}{I^{p}+I_{0}^{p}},
$$

where $I_{0}>0$ (Easland \& Wasserman, 1979; Hemila,
1987; Naka \& Rushton, 1966). Many neural network models (e.g., Hinton \& Sejnowski, 1986) employ the logistic function,

$$
a(I)=\frac{1}{1+\exp \left[p\left(I-I_{0}\right)\right]} .
$$

Each of the three candidate equations was evaluated in the additive and the multiplicative models.

\section{Categorical Outputs}

In Schweickert's (1985) model for speed-accuracy tradeoffs in discrete systems, each process is assumed to produce an output, which can be classified as correct or incorrect, for the input it was given. The probability that the response is correct is assumed to be the product of the probabilities that each individual process is correct. This product rule has also been proposed by Whitely and Schneider (1980) for correct responses to items in aptitude tests.

If certain other assumptions are met, then experimental factors affecting different processes would have additive effects not only on reaction time, but also on log percent correct (Schweickert, 1985). In other words, one can test whether factors have additive effects on accuracy (i.e., log percent correct) as well as on reaction time.

If the processes are in series, but their outputs do not meet the assumptions of Schweickert's (1985) model, one would expect the factors to have additive effects on reaction time, but not on log percent correct.

Changing the number of alternatives from two to four also changes the guessing rate from $1 / 2$ to $1 / 4$. One might wonder whether a correction for guessing is needed before testing for additivity. It turns out that since the correction appropriate for this factor would be the same at every level of the other factor, it would cancel out of the interaction, so the correction is not necessary.

An experiment was done to investigate the effects of intensity and number of alternatives in a hue identification task; a second experiment was done using lower intensities, with a payoff emphasizing speed to increase the error rates.

\section{EXPERIMENT 1}

\section{Method}

Subjects. The subjects were 18 Purdue University undergraduates who fulfilled a course requirement by participating. They were tested individually in four sessions of 90 min each. The first session was practice.

Stimuli. A Sony trinitron television screen attached to an Apple II computer with the modifications of Reed (1979) was placed on a table about $130 \mathrm{~cm}$ from the subject's eyes. The stimuli were horizontal bars, $4.4 \times 1.0 \mathrm{~cm}$, of red, orange, green, or blue. The Munsell values of the colors, without the filters used to change intensity, were approximately as follows: red, $10 \mathrm{RP} 7 / 8$; orange, 5 YR 7/8; green, 10 GY 6/10; blue, 5 PB 5/10 (Munsell, 1967).

Three Kodak neutral density Wratten filters (n.d. .30, .90,1.0) were used to manipulate intensity. The filters were mounted in manila mats that covered the screen except for the colored bar and 
a fixation dot. The .30 n.d. filter with a white tissue-paper backing produced the brightest level; the $1.0 \mathrm{n} . \mathrm{d}$. filter with the white tissue-paper backing produced the second brightest level; the .90 and 1.0 n.d. filters together $(1.9$ n.d.) with the white tissue-paper backing produced the dimmest level.

Glare was reduced by dimming the room lights and placing the screen in shadow. A Tektronix $\mathrm{J} 65231^{\circ}$ narrow-angle luminance probe mounted on a Tektronix $\mathrm{J} 16$ digital photometer was used to measure luminance. The luminance of the blank background screen was $7 \mathrm{~cd} / \mathrm{m}^{2}$. In the brightest condition, the luminances of the color patches, in $\mathrm{cd} / \mathrm{m}^{2}$ were: red, 29.19 ; orange, 36.63 ; green, 28.80 ; blue, 19.58. It was not feasible to eliminate the small differences in luminance.

Design and Procedure. At the start of each trial, a fixation point appeared for $1 \mathrm{sec}$ about $.5 \mathrm{~cm}$ below where the stimulus would appear. Upon termination of the point, one of the colored bars appeared for $100 \mathrm{msec}$.

The subject pressed one of the keys on the keyboard using his or her dominant hand to indicate which stimulus appeared. The keys were $\mathrm{v}, \mathrm{b}, \mathrm{n}$, and $\mathrm{m}$, for red, orange, green, and blue, respectively.

In half of the blocks of trials, the stimuli were selected at random from two alternative hues, and in the other half, all four alternatives were possible. The six stimulus conditions consisted of the three intensity levels combined with either two or four hue alternatives.

The 18 subjects were divided into six groups of 3 subjects, with members of each group receiving the same order of conditions and the same sequences of stimuli in their sessions. Each subject served for four sessions.

Each session was divided into 12 blocks. Each condition was presented once in the first 6 blocks and once in the second. Each of the groups received the conditions in a different order. One 6 $\times 6$ Latin square was used to determine the order for the first 6 blocks of the session, and another was used for the second 6 . Each block consisted of 40 trials at the same intensity. The first 8 were practice. Each of the alternative hues for a block occurred equally often, at random, in both the practice trials and the test trials.

For the conditions with two alternatives, each group of subjects encountered the 6 possible pairs of two colors once in each session. Over the six groups of subjects, each pair was used twice with each intensity level in each session.

A the start of the experiment, the subject was shown all of the hues, in each intensity condition. At the start of each block, the subject was told what the alternative hues would be for the block.

At the end of each block of trials, the subject was told his or her mean reaction time for the block and the number of errors. Small candies were used as payment. The subject began each session with 200 of them. At the end of each block, the subject lost 3 candies for every .1 sec in mean reaction time for correct test trials, and an additional $1 / 2$ for each error.

\section{Results and Discussion}

The means and standard deviations of the reaction times for correct and incorrect trials, and the percentage of errors are given in Tables 1 and 2.

Table 1

Experiment 1, Correct Trials: Means and Standard Deviations of Reaction Times, in Milliseconds

\begin{tabular}{|c|c|c|c|c|}
\hline \multirow{3}{*}{$\begin{array}{l}\text { Intensity } \\
\left(\mathrm{cd} / \mathrm{m}^{2}\right)\end{array}$} & \multicolumn{4}{|c|}{ Number of Alternatives } \\
\hline & \multicolumn{2}{|c|}{2} & \multicolumn{2}{|c|}{4} \\
\hline & $M$ & $S D$ & $\bar{M}$ & $S D$ \\
\hline 28.55 & 377 & 96 & 527 & 126 \\
\hline 5.70 & 396 & 96 & 538 & 122 \\
\hline .72 & 455 & 121 & 612 & 143 \\
\hline
\end{tabular}

Table 2

Experiment 1, Error Trials: Means and Standard Deviations of Reaction Times, in Milliseconds, and Percent Errors

\begin{tabular}{|c|c|c|c|c|c|c|}
\hline \multirow{3}{*}{$\begin{array}{c}\text { Intensity } \\
\left(\mathrm{cd} / \mathrm{m}^{2}\right)\end{array}$} & \multicolumn{6}{|c|}{ Number of Alternatives } \\
\hline & \multicolumn{3}{|c|}{2} & \multicolumn{3}{|c|}{9} \\
\hline & $M$ & $S D$ & $\mathrm{PE}$ & $M$ & $S D$ & PE \\
\hline 28.55 & 323 & 94 & 4.8 & 507 & 188 & 6.2 \\
\hline 5.70 & 334 & 98 & 4.1 & 527 & 139 & 6.3 \\
\hline .72 & 461 & 170 & 4.8 & 638 & 247 & 8.0 \\
\hline
\end{tabular}

Correct responses. An analysis of variance (ANOVA) was conducted, using the mean reaction time for the correct trials in each block. The factors were intensity, number of alternatives, position of the block within the session, group, subjects, and day, with subjects considered a random factor. Since certain effects are confounded in the Latin square design, interactions involving elements from two or more of the following sets were assumed to be zero: (1) intensity, number of alternatives, (2) groups, subjects, and (3) position.

There was a significant effect of intensity $[F(2,576)=$ 125.44, $p<.001]$ and of number of alternatives $[F(1,576)=1115.79, p<.001]$. There was, however, no interaction between them $[F(2,576)=.89]$.

The absence of an interaction between intensity and number of alternatives supports the idea that intensity and the number of alternatives affect different serial processes, and argues against the idea that intensity affects the rate of decision making.

The significant nuisance effects were group $[F(5,576)=$ $61.83, p<.001]$, subjects $[F(2,576)=24.71$, $p<.001]$, group $\times$ day $[F(10,576)=14.30$, $p<.001]$, and subjects $\times$ day $[F(4,576)=2.83$, $p<.05]$.

When an additive model is fit to the response times in Table 1, the residual sum of squares is 56.31 , and the total sum of squares about the mean is $41,067.16$, so $99.86 \%$ of the variance is accounted for. Nonetheless, the multiplicative model has a smaller residual. To fit the multiplicative model, the parameters of Equation 1 were estimated with the SAS nonlinear regression procedure. The estimates were $r=-749.57, a_{1}=.885, a_{2}=.875, a_{3}=$ $.830, b_{1}=1,000$, and $b_{2}=1,129$. One of the criterion values can be set arbitrarily, so $b_{1}$ was set to 1,000 . The residual sum of squares was 25.74 , an improvement over the additive model.

The base time $r$ is negative, and the following consideration might at first seem to make this possible. Laming (1968) suggested that subjects might begin sampling evidence from the display shortly before the stimulus actually appears. Would this lead to a negative base time, since timing starts at 0 with stimulus onset?-Let $s$ be the duration of the prestimulus sampling, and let $a_{0}$ be the rate of accrual of (irrelevant) evidence prior to stimulus onset. Let $d_{i j}$ be the decision time and let $r$ be the remaining processing. Then evidence will reach criterion, when

$$
b_{j}=a_{0} s+a_{i} d_{i j} \text {. }
$$

Then the total processing time will be $s+d_{i j}+r$, and 
the time from stimulus onset until the response will be

$$
t_{i j}=\left(b_{j}-a_{0} s\right) / a_{i}+r .
$$

This equation has the same form as Equation 1,

$$
t_{i j}=b_{j}^{*} / a_{i}+r
$$

where $b_{j}{ }^{*}=b_{j}-a_{0} s$, and $r$ remains positive. This reasoning cannot explain the negative estimate for the parameter $r$.

To summarize, fitting the additive model requires calculation of the row totals and one of the column totals (the other column total found by subtraction), so it has four parameters. The multiplicative model fits better, but has one more parameter, and an implausible estimated value for $r$.

The candidate equations for the accrual functions will be evaluated for both experiments together.

Errors. An ANOVA was conducted on log percent correct, using the same factors as for the correct response times. Note that the natural log of probability correct is approximately equal to minus the error probability, when the error probabilities are small, so this analysis is approximately equivalant to an ANOVA on the errors. There was an effect of number of alternatives $[F(1,576)=$ 26.18, $p<.001]$. There was no effect of intensity $[F(2,576)=2.35]$ and no interaction between them $[F(2$, $576)=1.22]$.

The significant nuisance effects were group $[F(5,576)$ $=13.98, p<.001]$ and subjects $[F(2,576)=27.28$, $p<.001]$.

These data are consistent with the model of Schweickert (1985), because there is no interaction; but the data do not provide a good test of the model, since one of the factors produced no main effect.

Error reaction times. Since the errors are few and distributed irregularly throughout the conditions, a statistical analysis of the reaction times on error trials would be cumbersome and not necessarily informative; but there is a noteworthy pattern. The error reaction times were faster than the correct reaction times when the intensities were at the highest two levels, and this ordering was reversed for the lowest intensity level. This is inconsistent with a rule of thumb proposed by Swensson (1972): error responses are faster than correct responses when the stimuli are easily discriminable and when speed is important (see Luce, 1986, p. 233, for a review).

More analytically, according to the continuous flow model (Coles, Gratton, Bashore, Eriksen, \& Donchin, 1985; Eriksen \& Schultz, 1979), there is an output from the encoding process for each response alternative. The rate of accrual of evidence would presumably be different for the correct alternative and the other alternatives. This can explain the result that error responses were faster than correct responses when the stimuli were bright, while the reverse held for dim stimuli.

Suppose, for illustration, that the accrual rate is a power function, $a(I)=I^{p}$, for the correct and incorrect alternatives. Suppose the value of $p$ is greater for an erroneous alternative than for the correct one. Then the curve for accrual rate as a function of intensity for the correct alternative will cross the curve for incorrect alternatives at some point. For intensities below this crossing point, correct responses will be faster than errors; above this point, the reverse will occur.

The next experiment was similar to the first, but in order to produce more errors, a wider range of intensities was used and the payoff emphasized speed.

\section{EXPERIMENT 2}

\section{Method}

Experiment 2 was carried out in the same way as Experiment 1, with the following changes.

For the three intensity levels, the neutral density filters used were .5 n.d.; 2.5 n.d.; and 3.3 n.d. No tissue-paper backing was used. With the $.5 \mathrm{n} . d$. filter, the luminances of the color patches, in $\mathrm{cd} / \mathrm{m}^{2}$, were red, 26.73; orange, 33.00; green, 26.62; blue, 19.60 .

The subjects were paid $\$ 3.50$ per hour. In addition there was a monetary payoff scheme. Each subject started each session with $\$ 10$. At the end of each block, the subject lost 5 cents for every $.1 \mathrm{sec}$ in average reaction time for correct test trials and $1 / 2$ cent for each error.

There were 6 subjects, who served for nine sessions. The first three sessions were practice, although the subjects were not informed of this. Each session consisted of 18 blocks. Three $6 \times 6$ Latin squares were used for each subject to assign the conditions to the blocks. Each of the six conditions occurred once in each third of the session. For the two alternative conditions, each of the six possible pairs of hues was used equally often with each intensity level when counted over all subjects.

\section{Results and Discussion}

The means and standard deviations of the reaction times for the correct and erroneous trials, as well as the error rates, are given in Tables 3 and 4 . Subjects found the dimmest level quite difficult, as is evident from the large error rates. Some subjects were dropped after the 3 practice days because their error rates were still close to chance for this intensity level.

For analysis, each session was divided into three parts: the first six blocks, the second six, and the third six. During each part, each combination of intensity and number of alternatives was presented once. An ANOVA was conducted on the mean reaction times for correct trials in each block, with the following factors: intensity, number of alternatives, part, day, and subjects, with subjects considered a random factor. Certain effects are confounded in the Latin square design, so interactions involving terms from two or more of the following sets were assumed to be zero: (1) intensity, number of alternatives; (2) subjects; (3) part.

Correct reaction times. There were significant effects of intensity $[F(2,570)=210.55, p<.001]$ and of number of alternatives $[F(1,570)=1291.30, p<.001]$. The interaction between them was not significant $[F(2,570)$ $=1.53]$.

There were also significant effects of subjects $[F(5,570)$ $=155.05, p<.001]$, and subjects $\times$ day $[F(25,570)=$ $3.15, p<.01]$. No other effects were significant. 
When the model stating that intensity and number of alternatives have additive effects on response time is fit to the correct response times in Table 3, the residual sum of squares is 64.33 , while the total sum of squares about the mean is $31,523.19$, so $99.79 \%$ of the variance is accounted for.

But, as in the first experiment, the multiplicative model in Equation 1 does better, in the sense that its residual sum of squares is smaller, .15. The parameter estimates for the model in Equation 1 are $r=-277.22, a_{1}=$ 1.566, $a_{2}=1.494, a_{3}=1.392, b_{1}=1,000$, and $b_{2}=$ 1,186. As in Experiment 1, the value of $b_{1}$ was arbitrarily set to 1,000 . The multiplicative model gives a much better fit, but it has one more free parameter than the additive model and, more importantly, produces an implausible negative estimate for the base time.

Errors. An analysis of variance using the same factors as for reaction time was carried out using log percent correct in each block. There were significant effects of intensity $[F(2,570)=330.37, p<.001]$; number of alternatives $[F(1,570)=94.85, p<.001]$; and their interaction $[F(2,570)=88.71, p<.001]$.

There were also significant effects of subjects $[F(5,570)$ $=34.84, p<.001]$ and of subjects $\times$ day $[F(25,570)$ $=1.94, p<.01$ ]. No other effects were significant.

The interaction between intensity and number of alternatives indicates that one of the assumptions of the model in Schweickert (1985) is wrong. In that model, after a process is completed, its output is either correct or incorrect, given the input.

Perhaps the model does not apply in this situation because the process affected by intensity does not produce a single ouput, but rather an accumulation of evidence for each response alternative (cf. Eriksen \& Schultz, 1979; Vickers, Burt, et al., 1985). For example, when the red stimulus appears, perhaps evidence accrues not only for the "red" response, but for the "orange" and other responses also. If the process affected by intensity produced a single output, then that output would be, presumably, sufficient for selecting a response. It is not clear what the role of the process affected by the number of alternatives would be.

Error reaction times. In Table 4 the error reaction times are numerically faster than the correct reaction times. The pattern is complicated because there seems to be little difference between correct and erroneous response

Table 3

Experiment 2, Correct Trials: Means and Standard Deviations of Reaction Times, in Milliseconds

\begin{tabular}{|c|c|c|c|c|}
\hline \multirow{3}{*}{$\begin{array}{l}\text { Intensity } \\
\left(\mathrm{cd} / \mathrm{m}^{2}\right)\end{array}$} & \multicolumn{4}{|c|}{ Number of Alternatives } \\
\hline & \multicolumn{2}{|c|}{2} & \multicolumn{2}{|c|}{4} \\
\hline & $M$ & $S D$ & $M$ & $S D$ \\
\hline 26.49 & 362 & 92 & 480 & 94 \\
\hline .26 & 392 & 89 & 517 & 96 \\
\hline .04 & 441 & 125 & 575 & 126 \\
\hline
\end{tabular}

Table 4

Experiment 2, Error Trials: Means and Standard Deviations of Reacton Times, in Milliseconds, and Percent Errors

\begin{tabular}{|c|c|c|c|c|c|c|}
\hline \multirow{3}{*}{$\begin{array}{r}\text { Intensity } \\
\left(\mathrm{cd} / \mathrm{m}^{2}\right)\end{array}$} & \multicolumn{6}{|c|}{ Number of Alternatives } \\
\hline & \multicolumn{3}{|c|}{2} & \multicolumn{3}{|c|}{4} \\
\hline & $M$ & $S D$ & PE & $M$ & $S D$ & $\mathrm{PE}$ \\
\hline 26.49 & 311 & 137 & 9.9 & 478 & 134 & 8.7 \\
\hline .26 & 360 & 96 & 10.7 & 490 & 122 & 12.5 \\
\hline .04 & 417 & 132 & 19.9 & 571 & 146 & 38.2 \\
\hline
\end{tabular}

times when there are four alternatives, while the difference is pronounced when there are two alternatives.

\section{COMPARISON OF ACCRUAL FUNCTIONS}

For each of the two experiments, the multiplicative model gave a better account of the correct response time data than the additive model, but with one more parameter. To put the models on a more equal footing, the accrual rate was predicted from intensity, 1 , by using the power, logistic, and Michaelis functions in Equations 2, 3 and 4, respectively. Each of these has two parameters, $I_{0}$ and $p$. The predicted value of $a(I)$ was used in the following equations for the additive and multiplicative models, respectively,

$$
t_{i j k}=r_{k} / a(I)+b_{j k}
$$

and

$$
t_{i j k}=r_{k}+b_{j k} / a(I),
$$

where $t_{i j k}$ is the expected reaction time in the condition with intensity level $i$, number of alternatives level $j$, in experiment number $k$.

Although each model has the same number of parameters, the parameters cannot be separately estimated in some models, so the residual degrees of freedom are not the same for all the models. For example, if $I_{0}=0$ in the power function, then the power function is equivalent to the Michaelis function in the additive model, as formulated above. To see this, note that the Michaelis equation in the additive model is

$$
\begin{aligned}
t_{i j k} & =r_{k}\left(I^{P}+I_{0}{ }^{p}\right) / I^{P}+b_{j k} \\
& =r_{k}{ }^{*} I^{P}+b_{j k}{ }^{*},
\end{aligned}
$$

where $r_{k}{ }^{*}=I_{0}{ }^{p} r_{k}$ and $b_{j k}{ }^{*}=r_{k}+b_{j k}$. For this reason, the additive model with the Michaelis function has one more degree of freedom than the additive model with the power function. Similarly, the logistic function yields one more degree of freedom in the additive model than in the multiplicative model.

The parameters, estimated with the SAS nonlinear regression procedure, may be found in Table 5 with the residual sums of squares for the various models. The percentage of variance accounted for by a model can be found by dividing its residual sum of squares by the total sum of squares about the mean, 74,176.10. Further statistical 
Table 5

Parameter Estimates and Residual Sums of Squares for Candidate Accrual Functions

\begin{tabular}{lrrrrrrrrrr}
\hline Functions & $r_{1}$ & $r_{2}$ & $p$ & \multicolumn{1}{c}{$I_{0}$} & $b_{11}$ & $b_{21}$ & $b_{12}$ & $b_{22}$ & $S S$. & $d f$ \\
\hline \multicolumn{8}{c}{} & \multicolumn{8}{c}{ Additive Models } \\
Logistic & 141 & 9 & 4.29 & .57 & 243 & 392 & 349 & 475 & 315.55 & 5 \\
Power & 72 & 15 & .70 & -.04 & 370 & 520 & 357 & 482 & 109.53 & 4 \\
Michaelis & 67 & 14 & .52 & 1.47 & 295 & 444 & 342 & 467 & 134.74 & 5 \\
\multicolumn{8}{c}{ Multiplicative Models } \\
Logistic & 245 & 324 & 1.50 & -.05 & 150 & 285 & 51 & 131 & 2523.94 & 4 \\
Power & -1358 & -150 & .01 & .04 & 1801 & 1953 & 533 & 655 & 589.71 & 4 \\
Michaelis & -2503 & -209 & .51 & .028 & 2866 & 3013 & 569 & 686 & 62.62 & 4 \\
\hline
\end{tabular}

comparisons of the models are not feasible because the distributions of the estimates are unknown due to the iterative estimation procedure.

Evidently, the logistic function is not suitable because of its large residual sum of squares. The power function gives the best fit for the additive models, and the Michaelis function does so for the multiplicative models. An argument against the Michaelis equation in the multiplicative model is that the base time is estimated to be a negative number, longer even than the warning interval. Nonetheless, this model gives the best fit to the data.

\section{DISCUSSION}

In a review of the effects of intensity on reaction time, Nissen (1977) concluded that in choice-reaction time tasks, several manipulations expected to affect the criterion did not produce the overadditive interaction with intensity predicted by Grice's (1968) model, Equation 1. Here too, the evidence suggests that the additive model is closer to the correct account than the multiplicative model is. If variable sampling models are correct, then evidently intensity does not affect the accrual rate, while the number of alternatives affects the criterion.

For the accrual functions, if the additive model is valid, the power function originally suggested by Piéron for simple reaction times also accords with choice reaction times. The Michaelis function gives a good account of the data for both models.

Analysis of log percent correct rejected Schweickert's (1985) model for these data. The model's key assumption is that each process produces an output that is correct or incorrect for its given input, and that the probability of a correct response is the product of these conditional probabilities. Experiments supporting that model used words, characters, and arrows as stimuli (Schweickert, 1985). Perhaps these stimuli elicit categorical processing, whereas colors do not. With colors, some evidence may accrue for each color alternative when a particular stimulus is presented; that is, the process affected by intensity may not produce a single output, but rather several. An experiment by Coles et al. (1985), in which more than one letter was presented per trial, suggests that evidence for an erroneous alternative sometimes permeates through the system all the way to response ini- tiation. Such systems require a different model from that of Schweickert (1985).

Ordinarily, with the additive factor method one does not learn the durations of the processing stages. Here, if the power function in the additive model is true, we can calculate the time spent in encoding by subtracting the parameter $b_{j k}$ from the reaction time. In the first experiment, encoding ranges from $7 \mathrm{msec}$ at the brightest level to $85 \mathrm{msec}$ at the dimmest, when there are two alternatives. This indicates that the processing affected by intensity can be over very early.

\section{REFERENCES}

Brebner, J. M. T., \& Welford, A. T. (1980). Introduction: An historical background sketch. In A. T. Welford (Ed.), Reaction times (pp. 124). New York: Academic Press.

BuSEMEYER, J. R., \& JoNES, L. E. (1983). Analysis of multiplicative combination rules when the causal variables are measured with error. Psychological Bulletin, 93, 549-562.

Coles, M. G., Gratton, G., Bashore, T. R., Eriksen, C. W., \& DonCHIN, E. (1985). A psychophysical investigation of the continuous flow model of human information processing. Journal of Experimental Psychology: Human Perception \& Performance, 11, 529-553.

Easland, G. S., Wasserman, G. S. (1979). Multiple intracellular contributions to light adaptation in limulus ommatidia. Vision Research, 19, 1-8.

ERIKSEN, C. W., \& SCHULTZ, D. W. (1979). Information processing in visual search: A continuous flow conception and experimental results. Perception \& Psychophysics, 25, 249-263.

EveretT, B. L., HochHaus, L., \& Brown, J. R. (1985). Letter-naming as a function of intensity, degradation, S-R compatibility, and practice. Perception \& Psychophysics, 37, 467-470.

Fisher, D. L. , \& GoldsteIN, W. M. (1983). Stochastic PERT networks as models of cognition: Derivation of mean, variance, and distribution of reaction time using order-of-processing (OP) diagrams. Journal of Mathematical Psychology, 27, 121-151.

GARNER, W. R. (1974). The processing of information and structure. Potomac, MD: Halstead.

Grice, G. R. (1968). Stimulus intensity and response evocation. Psychological Review, 75, 359-373.

Hemila, S. (1987). The stimulus-response functions of visual systems. Vision Research, 27, 1253-1261.

Hinton, G. E., \& Sejnowski, T. J. (1986). Learning and relearning in Boltzmann machines. In D. E. Rumelhart \& J. L. McClelland (Eds.), Parallel distributed processing: Explorations in the microstructure of cognition (pp. 282-317). Cambridge, MA: MIT Press.

Kohfeld, D. L., SANTeE, J. L., \& Wallace, N. D. (1981). Loudness and reaction time: I. Perception \& Psychophysics, 29, 535-549.

LAMING, D. R. J. (1968). Information theory of choice-reaction times. London: Academic Press. 
LiNk, S. W. (1979). Improvements on a new model for choice reaction time. Perception \& Psychophysics, 25, 443-446.

LUCE, R. D. (1986). Response times: Their role in inferring elementary mental organization. New York: Oxford University Press.

McGill, W. J. (1963). Stochastic latency mechanisms. In R. D. Luce, R. R. Bush, \& E. Galanter (Eds.), Handbook of mathematical psychology (Vol. 1, pp. 309-360). New York: Wiley.

Munsell Color Company, Inc. (1967). Munsell book of color. Baltimore, MD: Author.

NAKA, K. I., \& RUShton, W. A. (1966). S-potentials from luminosity units in the retina of fish (Cyprinidae). Journal of Physiology, 185, 587-599.

Niemi, P. (1979). Stimulus intensity effects on auditory and visual reaction processes. Acta Psychologica, 43, 299-312.

Nissen, M. J. (1977). Stimulus intensity and information processing. Perception \& Psychophysics, 22, 338-352.

Piéron, H. (1914). Recherches sur les lois de variation des temps de latence sensorielle en fonction des intensités excitatrices. L'Année Psychologique, 20, 17-96.

Préron, H. (1920). Nouvelles recherches sur l'analyse du temps de latence sensorielle et sur la loi qui relie le temps à l'intensité d'excitation. L'Année Psychologique, 22, 58-142.

POSNER, M. I. (1978). Chronometric explorations of mind. Hillsdale, NJ: Erlbaum.

RaAb, D., Fehrer, E., \& Hershenson, M. (1961). Visual RT and the Broca-Sulzer phenomenon. Journal of Experimental Psychology, 61, 193-199.

ReED, A. V. (1979). Microcomputer display timing: Problems and solutions. Behavior Research Methods \& Instrumentation, 11, 572-576.

SANDERS, A. F., ANDriessen, J. E. B. (1978). A suppressing effect of response selection on immediate arousal in a choice reaction task. Acta Psychologica, 42, 181-186.
SCHWEICKERT, R. (1978). A critical path generalization of the additive factor method: Analysis of a Stroop task. Journal of Mathematical Psychology, 18, 105-139.

SCHWEICKERT, R. (1985). Separable effects of factors on speed and accuracy: Memory scanning, lexical decision, and choice tasks. Psychological Bulletin, 97, 530-546.

Shwartz, S. P., Pombrantz, J. R., \& Egeth, H. (1977). State and process limitations in information processing: An additive factor analysis. Joumal of Experimental Psychology: Human Perception \& Performance, 3, 402-410.

SMITH, M. C. (1967). Reaction time to a second stimulus as a function of intensity of the first stimulus. Quarterly Journal of Experimental Psychology, 19, 125-132.

STERNBERG, S. (1969). The discovery of processing stages: Extensions of Donders' method. Acta Psychologica, 30, 276-315.

SWENSSON, R. G. (1972). The elusive tradeoff: Speed versus accuracy in visual discrimination tasks. Perception \& Psychophysics, 12, 16-32.

TownsEnd, J. T., A AshBY, F. G. (1983). The stochastic modeling of elementary psychological processes. Cambridge: Cambridge University Press.

Vickers, D., Burt, J., Smith, P., \& Brown, M. (1985). Experimental paradigms emphasizing state or process limitations: 1 . Effects on speed-accuracy tradeoffs. Acta Psychologica, 59, 129-161.

Vickers, D., Smith, P., BurT, J., \& Brown, M. (1985). Experimental paradigms emphasizing state or process limitations: II. Effects on confidence. Acta Psychologica, 59, 163-193.

Whitely, S. E., Schneider, L. (1980). Process outcome madels for verbal aptitude. (Tech. Rep. NIE-80-1). Lawrence: University of Kansas.

(Manuscript received November 23, 1987; revision accepted for publication May 6, 1988.) 